\title{
A transvaginal endoscopy-based technique for performing ovarian examinations in sows
}

\author{
Minami W OKUYAMA ${ }^{1-3)}$, Tomochika SUGIURA ${ }^{1)}$, Masaharu MORIYOSHI ${ }^{1}$, \\ Kazuto YAMASHITA ${ }^{4)}$, Jun TAMURA ${ }^{4)}$ and Seiji KATAGIRI ${ }^{1,5)}$ \\ 1) Department of Large Animal Clinical Sciences, School of Veterinary Medicine, Rakuno Gakuen University, \\ Ebetsu 069-8501, Japan \\ ${ }^{2)}$ Department of Veterinary Clinical Sciences, Graduate School of Veterinary Medicine, Hokkaido University, \\ Sapporo 060-0818, Japan \\ 3) Faculty of Medicine, Oita University, Oita 879-5593, Japan \\ 4) Department of Small Animal Clinical Sciences, School of Veterinary Medicine, Rakuno Gakuen University, \\ Ebetsu 069-8501, Japan \\ 5) Department of Environmental Veterinary Sciences, Graduate School of Veterinary Medicine, Hokkaido University, \\ Sapporo 060-0818, Japan
}

\begin{abstract}
For examining pig ovaries, which have complex structures, laparoscopy is a useful technique, but requires general anesthesia; therefore, it cannot be performed repeatedly within a short period of time. We report a transvaginal endoscopybased technique for conducting ovarian examinations without general anesthesia. Sows were sedated in pig stalls. Using a colonoscope, the vaginal wall was punctured with a trocar. To avoid the trocar being caught in the broad ligament of the uterus or the connective tissue around the vagina, the trocar was inserted close to the external uterine os and between the 2:00 and 3:00 or the 9:00 and 10:00 positions (in a clockwise direction). Then, a urethroscope was inserted into the abdomen, and an examination was carried out after the ovaries had been moved towards the urethroscope camera via rectal palpation. This less invasive procedure may allow repeated examinations and will increase our understanding of ovarian dynamics in pigs.
\end{abstract}

Key words: Ovarian observation, Sows, Transvaginal endoscopy

(J. Reprod. Dev. 63: 617-622, 2017)

$\mathbf{E}$ valuations of ovarian status are important for facilitating effective reproductive management in domestic animals. In pigs, high reproductive potential is characterized by a short open period and a large litter size. These characteristics are related in the structure of the pig ovary complex; for example, each ovary contains high numbers of follicles and/or corpus lutea (CL), which change in size rapidly. It is difficult to assess their numbers and states, and there are few tools for examining complex ovarian structures. Such technical limitations have adversely affected our knowledge of pig reproductive physiology.

As in other domestic animals, rectal palpation can be used to estimate the presence and numbers of follicles and CL; however, rectal palpation in pigs requires specific skills because of the difficulties caused by the long uterus horn and small pelvic cavity in pigs. Moreover, it is difficult to count the exact numbers of ovarian structures in pigs only by palpation. Therefore, ultrasonography (USG) is the first-choice method for examining the morphology of the ovary and can be used to monitor ovarian dynamics in a

Received: May 11, 2017

Accepted: September 25, 2017

Published online in J-STAGE: October 14, 2017

(C)2017 by the Society for Reproduction and Development

Correspondence: S Katagiri (e-mail: katagiri@vetmed.hokudai.ac.jp)

This is an open-access article distributed under the terms of the Creative Commons Attribution Non-Commercial No Derivatives (by-nc-nd) License. (CC-BY-NC-ND 4.0: https://creativecommons.org/licenses/by-nc-nd/4.0/) non-invasive manner [1, 2].

Ultrasonography can be used for measuring the sizes of follicles and CL and to facilitate ovum pick-up (OPU), as well as for measuring blood flow by using Doppler echography. However, USG cannot provide live color images, which can help scientists deal with more leading-edge technologies. For example, laparoscopy, which can provide live images, has also been used for studies of ovarian physiology [3, 4], diagnosis of reproductive disorders [5], and development of new reproductive technology (embryo transfer [6], oviductal insemination [7], OPU [8] and embryo collection from the reproductive tract [9]) in pigs.

In pigs, laparoscopy is performed by placing the animal on its back under general anesthesia; therefore, appropriate equipment, such as surgical beds and inhalation anesthesia apparatus, is required. Moreover, laparoscopy cannot be repeated within a short period since general anesthesia can cause severe stress and might result in alterations in the pig's physical functions. Thus, the value of laparoscopy would increase markedly if laparoscopic examinations could be performed in the standing position and without general anesthesia.

In cows, OPU, which is a technique used to retrieve oocytes from live cows, has been performed using transvaginal ultrasound scanning [10]. This transvaginal approach is usually conducted in the standing position under caudal epidural anesthesia or mild sedation, which makes repeated examinations of ovarian structures possible. Ferguson et al. reported that this approach can be performed in pigs for OPU [11]. Less invasive and repeatable laparoscopy 
can contribute to revealing ovarian dynamics more precisely such as neoangiogenesis or ovulation. Here, we report a transvaginal endoscopy-based technique for performing ovarian examinations in the standing position.

The present study was conducted according to the institutional guidelines for animal experiments of Rakuno Gakuen University (approval No. VH25C10). We used a total of 8 sows (body weight $[$ mean $\pm \mathrm{SD}$ ]: $221.7 \pm 14.4 \mathrm{~kg}$ ) in three studies. The first study aimed to develop instruments and protocols for use under inhalation anesthesia. The second study was designed to confirm that the developed procedure could be performed under sedation in the standing position. The third study examined the practicability of performing repeated examinations using the developed protocol.

In study 1, which aimed to develop instruments and protocols especially for use with a rigid urethroscope (A2942A; Olympus, Tokyo, Japan), we used 2 Large White sows (body weight: 203 and $227 \mathrm{~kg}$ ). Stainless steel outer guides and trocars were prepared for use during the transvaginal procedure (see Methods, Fig. 1), i.e., to puncture the vaginal wall from the vaginal cavity to the abdominal cavity. During standard laparoscopic examinations, pigs are placed in the supine position, and the abdominal cavity is insufflated with gas to broaden the viewing field and facilitate examination. However, this approach requires general anesthesia [3] and normally requires a special gas delivery device [12]. Instead, an inner guide was developed for our procedure to prevent the camera from coming into contact with the tissue. Pipes of two different lengths $(43.5$ and $44.0 \mathrm{~cm}$, respectively) were prepared. The lengths were determined to be 0.5 and $1 \mathrm{~cm}$ longer than the effective length $(43.0 \mathrm{~cm}$, Fig. 1A) of the urethroscope. The sows were kept under inhalation anesthesia (described in Methods) and placed in the prone position on a surgical air bed. For the examinations, a rigid urethroscope was inserted together with an inner guide pipe into the abdominal cavity. Then, the operator grasped the ovaries in their hand via the rectum and drew them into the visual field of the scope (Fig. 2, Supplemental movie 1: online only). The procedure took about $30 \mathrm{~min}$ from the induction of anesthesia. The $43.5-\mathrm{cm}$ inner guide provided a wider and larger viewing field than the 44-cm inner guide (Figs. 1B and C). At the end of each operation, the sows were euthanized, and the vaginal wall puncture site was examined anatomically.

In study 2, we used 2 hybrid sows (Large White $\times$ Landrace, body weight: 196 and $232 \mathrm{~kg}$, parity: 5 and 7, estrus cycle: 22 days in both sows) to confirm that the abovementioned procedures can be performed under sedation in the standing position. The sows were sedated (described in Methods) and pressed against one side of the stall (Fig. 3). The space between the pigs and the other side of the wall was filled with surgical mattresses $(105 \times 132 \times 10 \mathrm{~cm})$. To keep the sows in the standing position, small surgical mattresses $(50 \times 60$ $\times 20 \mathrm{~cm}$ and/or $30 \times 50 \times 12 \mathrm{~cm}$ ) were placed under the abdomen. An intravenous line was kept in the auricular vein in case supplemental anesthesia was needed. In one sow, the ovarian examination was completed under sedation using the procedure developed in study 1 . In the other sow, it took time to penetrate the vaginal wall because the trocar got caught in the broad ligament of the uterus; therefore, an attempt was made to penetrate the vaginal wall at another site. At the end of each operation, the sows were euthanized, and the vaginal wall puncture site was examined anatomically.
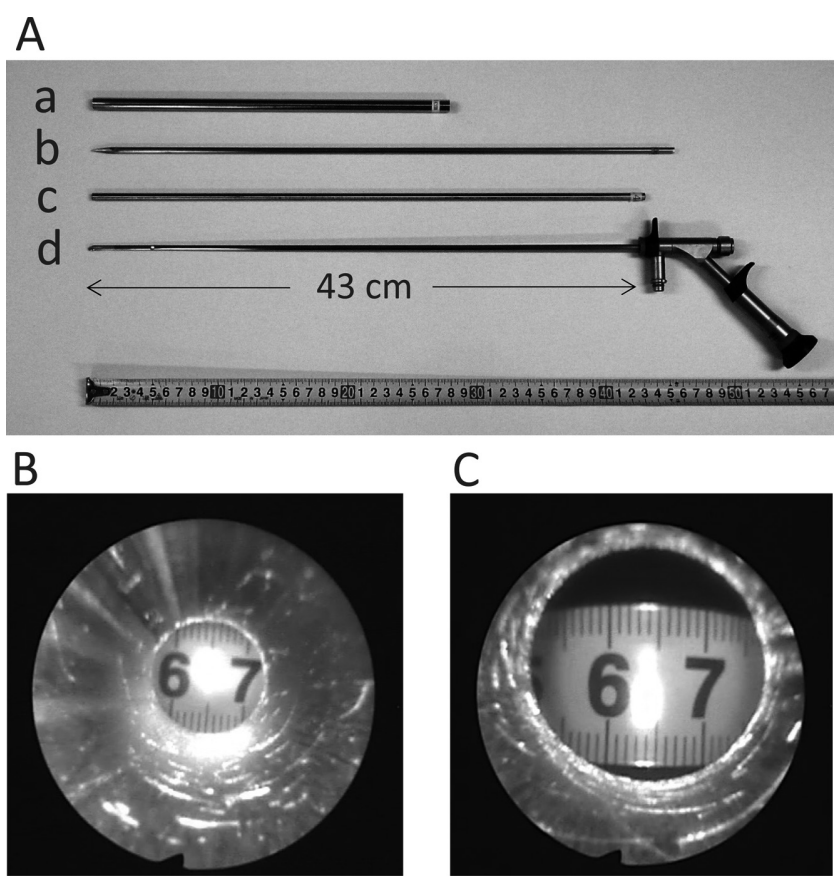

Fig. 1. A: Instruments used for the transvaginal endoscopic examinations a: Outer guide (inner diameter: $8.5 \mathrm{~mm}$, outer diameter: $9.5 \mathrm{~mm}$, length: $28 \mathrm{~cm}$ ), b: trocar (diameter: $5 \mathrm{~mm}$, length: $46 \mathrm{~cm}$ ), c: inner guide (inner diameter: $4.9 \mathrm{~mm}$, outer diameter: $6.0 \mathrm{~mm}$, length: $43.5 \mathrm{~cm}$ ), and d: urethroscope (A2942A; Olympus, Tokyo, Japan); $\mathrm{B}$ : The viewing field obtained with the $44.0-\mathrm{cm}$ inner guide; $\mathrm{C}$ : The viewing field obtained with the $43.5-\mathrm{cm}$ inner guide.

In study 3, we used 4 hybrid sows (Large White $\times$ Landrace, body weight: 196-227 kg, parity: 6-7, estrus cycle: 21-22 days) to examine the practicability of using the developed protocol to perform repeated examinations in a maintaining stall $(190 \times 58 \times$ $103 \mathrm{~cm}$ ) under sedation. Two examinations were conducted in each sow with a 2- or 3-day interval between them, 8 experiments in total. In some trials, the trocar got trapped in the broad ligament of the uterus (see trials 4-6 in Table 1), and puncturing of the vaginal wall failed. During detailed examinations of the vaginal wall puncture sites performed in trials $1-6$, we found that the trocar became trapped when it was inserted immediately lateral to or below the external os of the uterus (between the 3:00 and 5:00 or 7:00 and 9:00 positions in a clockwise direction from the external os). When the puncture site was located too caudal from the uterine os, the trocar sometimes became caught in the connective tissue around the vagina. Based on the results of the trials in study 3 , we concluded that the vaginal wall can be punctured smoothly by inserting the trocar near to the external os of the uterus and between the 2:00 and 3:00 or 9:00 and 10:00 positions (in a clockwise direction) (Fig. 4A). It took about $5-10$ min to puncture the vaginal wall. The success rate was $75 \%$ in trials $7-10$ with this improved method, although it was $25 \%$ in trials 3-6. Throughout the studies including 2 trials with luteal phase sows in study 1 , the ovarian status such as whether they were in the luteal or follicular phase did not affect the success of the puncture. In sow WL5, the vaginal wall could not be penetrated, even using this 
A

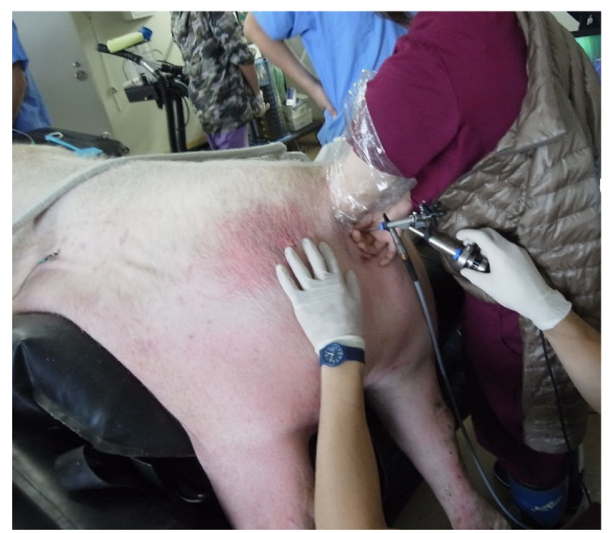

B

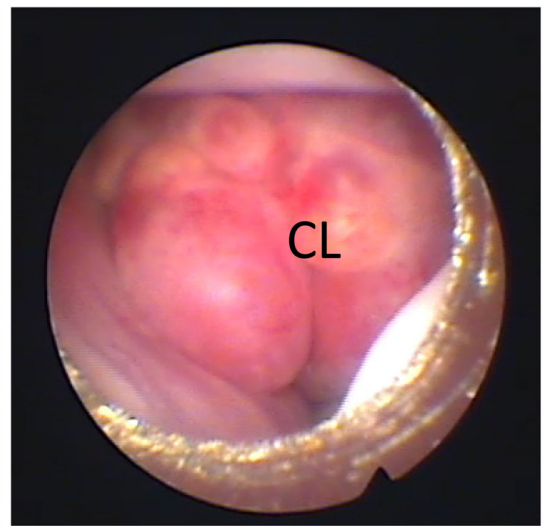

C

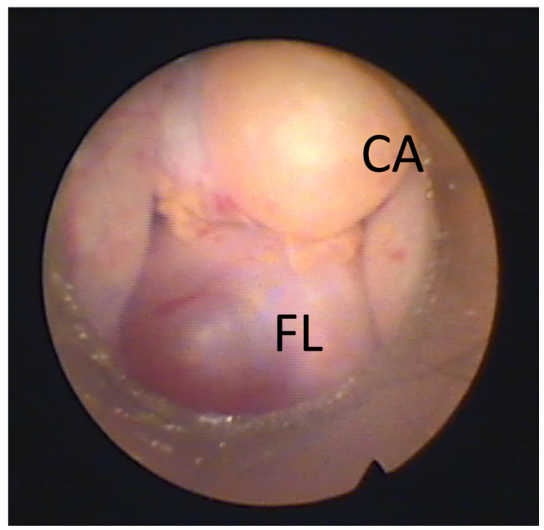

Fig. 2. Transvaginal endoscopic examination procedure and images of the ovaries obtained in experiment 1 . The operator grasped one of the ovaries through the rectum (A), and a ureteroscope was inserted transvaginally to view the ovaries (B, C). CL: corpus luteum, FL: follicle, CA: corpus albicans.
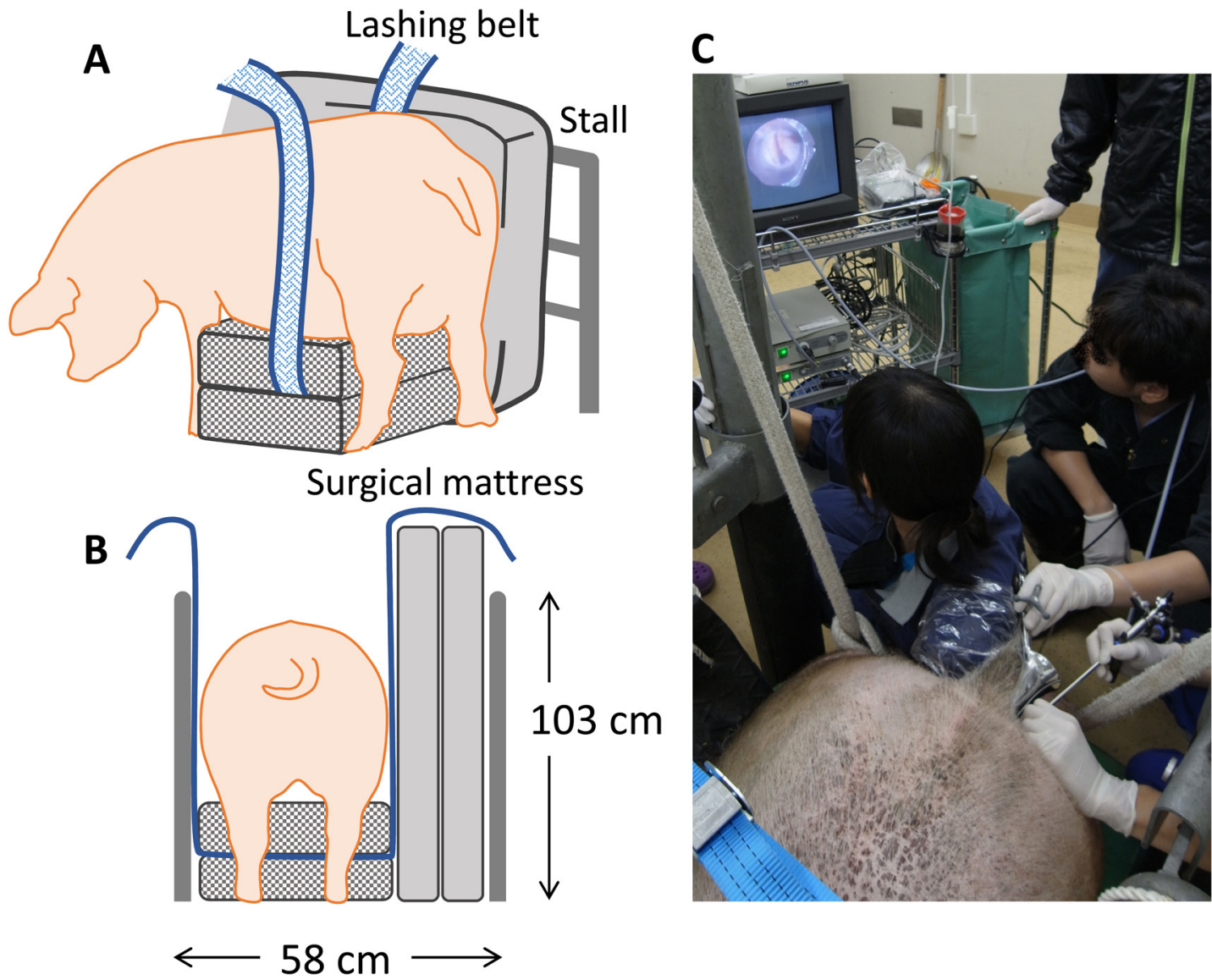

Fig. 3. Physical immobilization of the sow in a stall and transvaginal endoscopic examination of the ovaries. Sows under sedation were gently kept in position in a maintaining stall using a hard surgical mattress, and a lashing belt was used to sling up the sows to prevent them from lying down on the floor (A, B). Examinations were performed in an operation stall for large animals (C). 
Table I. Results of the transvaginal endoscopy examinations

\begin{tabular}{lcclcc}
\hline & Trials & Sow ID & Ovarian status & Puncturing of the vaginal wall & Ovaries observed \\
\hline Study 2 & 1 & WL6 & FL (Day -1) & Succeeded & Covered/Exposed \\
& 2 & WL3297 & FL (Day -1) & Succeeded * & Covered/Exposed \\
\hline Study 3 & 3 & WL3258 & FL (Day 0) & Succeeded & Covered/Exposed \\
& 4 & WL3258 & FL (Day 1) & Failed & - \\
& 5 & WL3 & CL (Day 10) & Failed & - \\
& 6 & WL5 & CL (Day 12) & Failed & Covered/Exposed \\
& 7 & WL4 & FL (Day -5) & Succeeded & Exposed \\
& 8 & WL3 & CL (Day 13) & Succeeded & - \\
& 9 & WL5 & CL (Day 15) & Failed & Covered \\
\hline
\end{tabular}

Ovarian status: The first day of estrus was set as Day 0. CL: Luteal phase, FL: Follicular phase. * Puncturing of the vaginal wall was successful after several failures. Covered: the ovaries were covered by the mesosalpinx and fimbria ovarica. Exposed: the ovaries were observed directly.

improved method. Necropsy showed that the vagina was torsioned near to its os.

After puncturing the vaginal wall, a transvaginal ovarian examination was successfully performed under sedation in the standing position using the procedure described in Fig. 4. The vaginal wall injuries caused by the instruments healed within 2 or 3 days. The ovaries were sometimes almost entirely covered by the mesosalpinx and fimbria ovarica (Table 1, Supplemental movie 2: online only). Of the 6 trials conducted in studies 2 and 3, the ovaries could only be directly observed in a single case (trial 8), and they were covered by the mesosalpinx and fimbria ovarica in the other 5 cases. In 4 of the 5 latter cases, the operator was able to manipulate the mesosalpinx and fimbria ovarica, then expose the ovaries by hand through the rectum. Although it is not clear when and how the mesosalpinx and fimbria ovarica covers the ovaries, it seems to often occur in the follicular phase. In such cases, instruments such as alligator forceps might be helpful for removing the mesosalpinx and fimbria ovarica. In the present study, we also attempted to obtain ovarian follicular liquid or luteal tissue using custom-made needles $(22 \mathrm{G} / 62 \mathrm{~cm}$ for follicles, 18 G/60 cm for CL; Misawa Medical Industry, Saitama, Japan) (Supplemental movie 3: online only). In each sampling procedure, although follicles or CL were punctured at 2-4 sites, the ovarian dynamics and follicular development proceeded according to normal cycles. Three to 6 days after the final operation, all the sows were euthanized and checked anatomically. There were few puncture scars on the ovaries and no evidence of adhesion. The puncture scars in the vaginal wall were also too small to identify, and there was no evidence of inflammation or adhesion.

In summary, we have developed a procedure for performing transvaginal endoscopic examinations of the ovaries in sows under sedation in the standing position. Although no methods for transvaginal ovarian sampling have been established yet, this procedure allows repeated examinations to be conducted and can be used to collect follicular and luteal samples. This procedure might contribute to increasing our understanding of ovarian dynamics in sows in a less invasive and less traumatic way.

\section{Methods}

\section{Instruments developed}

In experiment 1, we developed the following instruments: outer guides, trocars, and inner guides suitable for use with a rigid urethroscope (A2942A; Olympus, Tokyo, Japan) (Fig. 1A). All instruments were sterilized before the start of the experiment.

\section{Animal sedation, anesthesia, and euthanasia}

In experiment 1 , the sows were sedated via the intramuscular injection of a mixture of $40 \mu \mathrm{g} / \mathrm{kg}$ body weight (BW) medetomidine (Dorbene vet; Kyoritsu Seiyaku, Tokyo, Japan) and $0.2 \mathrm{mg} / \mathrm{kg} \mathrm{BW}$ midazolam (Dormicum; Astellas Pharma, Tokyo, Japan), and anesthesia was induced with an intravenous injection of $6 \mathrm{mg} / \mathrm{kg} \mathrm{BW}$ propofol (1\% propofol injection for animals; Mylan Seiyaku, Tokyo, Japan). Then, the sows were orotracheally intubated and maintained at a surgical depth of anesthesia with 2-4\% sevoflurane (SevoFlo; DS Pharma Animal Health, Osaka, Japan) in oxygen.

In experiments 2 and 3, the sows were sedated via the intramuscular injection of mafoprazine mesylate at a dose of $0.5 \mathrm{mg} /$ kg BW (Mafropane; DS Pharma Animal Health), followed by the intramuscular injection of a mixture of $40 \mu \mathrm{g} / \mathrm{kg}$ BW medetomidine and $0.2 \mathrm{mg} / \mathrm{kg}$ midazolam. To avoid peristalsis and contraction of the rectum and uterus, epidural anesthesia was induced with $3 \mathrm{ml}$ of $2 \%$ lidocaine (xylocaine; Astra-Zeneca, Osaka, Japan) or the intravenous injection of $1 \mathrm{ml}$ of butylscopolamine bromide (20 mg/ml Buscopan; Boehringer Ingelheim International $\mathrm{GmbH}$, Germany). In one sow in experiment 2 , the operation time was prolonged with supplemental anesthesia, which was induced via the intravenous injection of 2.5 $\mathrm{mg} / \mathrm{kg}$ BW sodium pentobarbital (Somnopentyl; Kyoritsu Seiyaku). After each operation in experiment 3, the sows were awakened using $0.2 \mathrm{mg} / \mathrm{ml}$ atipamezole (Atipame, Kyoritsu Seiyaku).

Euthanasia was achieved with an overdose of sodium pentobarbital under anesthesia, as described above.

\section{Transvaginal endoscopic examination procedure}

The sows were placed in pig stalls. After removing any feces, the pigs' external genitalia were washed and cleaned. The sows were 

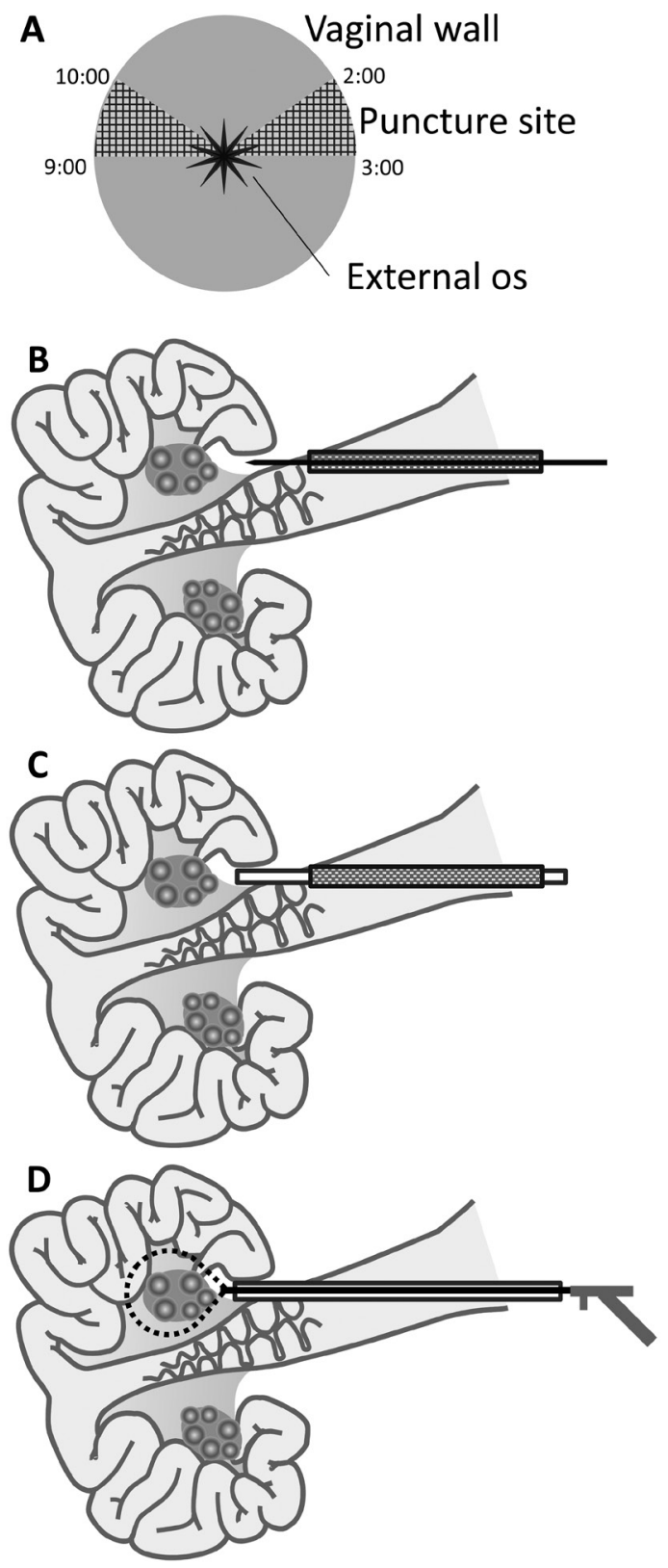

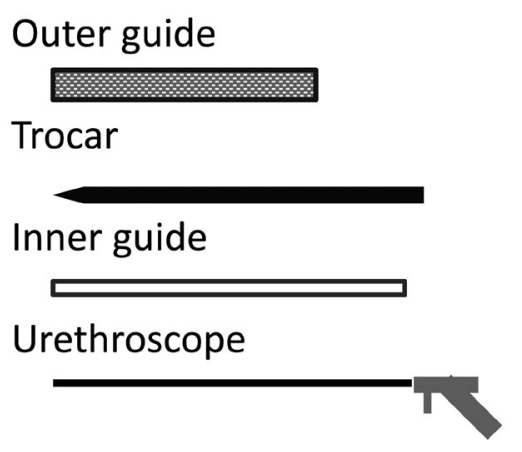

Fig. 4. Procedure for the transvaginal endoscopic examinations. The edge of the outer guide was placed on one side of the external os of the uterus. The puncture site (A) was located close to the external os of the uterus and between the 2:00 and 3:00 or the 9:00 and 10:00 positions (in a clockwise direction) (shaded areas). A trocar was inserted through the outer guide, and a puncture was made through the vaginal wall and into the abdominal cavity (B). The trocar was removed, and an inner guide was inserted into the abdominal cavity along with the outer guide $(\mathrm{C})$. The outer guide was removed and a urethroscope was inserted through the inner guide, and then an examination was performed (D). then sedated as described above (in the Animal sedation, anesthesia, and euthanasia [experiment 3] section of the Material and methods). To prevent the sows from lying down on the floor; i.e., to keep them standing, hard mattresses were placed around their bodies (Figs. 3A and B). A colonoscope was inserted into the vagina, and the vaginal wall was wiped with sterile gauze and iodized cotton $(0.5 \%$ povidone iodine). Local anesthesia was induced using $2 \%$ lidocaine jelly ( $2 \%$ xylocaine jelly, Astra-Zeneca, Osaka, Japan), and an outer guide was placed at the side of the external os of the uterus. The edge of the outer guide was pressed against the vaginal wall between the 2:00 and 3:00 or the 9:00 and 10:00 positions (in a clockwise direction from the external os) (Fig. 4A). Subsequently, a trocar was inserted in the outer guide, and a puncture was made through the vaginal wall and into the abdominal cavity (Fig. 4B). The trocar was removed, and an inner guide was inserted into the abdominal cavity along the outer guide (Fig. 4C). The operator moved the target ovary close to the edge of the inner guide via rectal palpation. A urethroscope was inserted through the inner guide, and an examination was performed (Figs. 3C and 4D) by flushing the urethroscope camera using warm sterilized saline. After the examination, the urethroscope and the inner guide were removed, and the vaginal lumen was cleaned using iodized cotton. The sows were awakened with an antagonist drug, as described above. 


\section{Acknowledgements}

We would like to thank all of the contributors at Rakuno Gakuen University, especially Dr Yamada, Mr Ozaki, and Dr Sugano of the Department of Sustainable Agriculture for their kind help in managing the sows. We also thank the students of the Laboratory of Theriogenology. Finally, we thank Mr Yasuhiro Misawa of Misawa Medical Industry Co., Ltd., for developing the needles used to collect the ovarian components.

This study was supported by the Science and Technology Research Promotion Program for Agriculture, Forestry, Fisheries, and Food Industry (25052C) of the Ministry of Agriculture, Forestry, and Fisheries of Japan.

\section{References}

1. Soede NM, Noordhuizen JP, Kemp B. The duration of ovulation in pigs, studied by transrectal ultrasonography, is not related to early embryonic diversity. Theriogenology 1992; 38: 653-666. [Medline] [CrossRef]

2. Schwarz T, Murawski M, Wierzchoś E, Bartlewski P. An ultrasonographic study of ovarian antral follicular dynamics in prepubertal gilts during the expected activation of the hypothalamo-pituitary-ovarian axis. J Reprod Dev 2013; 59: 409-414. [Medline] [CrossRef]

3. Wildt DE, Fujimoto S, Spencer JL, Dukelow WR. Direct ovarian observation in the pig by means of laparoscopy. J Reprod Fertil 1973; 35: 541-543. [Medline] [CrossRef]

4. Brüssow KP, Ratky J, Kanitz W, Becker F. Determination of the duration of ovulation in gilts by means of laparoscopy. Reprod Domest Anim 1990; 25: 184-190. [CrossRef]

5. Wildt DE, Morcom CB, Dukelow WR. Laparoscopic pregnancy diagnosis and uterine fluid recovery in swine. J Reprod Fertil 1975; 44: 301-304. [Medline] [CrossRef]

6. Hazeleger W, Kemp B. Recent developments in pig embryo transfer. Theriogenology 2001; 56: 1321-1331. [Medline] [CrossRef]

7. Morcom CB, Dukelow WR. A research technique for the oviductal insemination of pigs using laparoscopy. Lab Anim Sci 1980; 30: 1030-1031. [Medline]

8. Brüssow KP, Ratky J. Repeated laparoscopical follicular puncture and oocyte aspiration in swine. Reprod Domest Anim 1994; 29: 494-502. [CrossRef]

9. Brüssow KP, Ratky J. Endoscopic collection of porcine embryos. Reprod Domest Anim 1996; 31: 711-715. [CrossRef]

10. Pieterse MC, Vos PL, Kruip TA, Willemse AH, Taverne MA. Characteristics of bovine estrous cycles during repeated transvaginal, ultrasound-guided puncturing of follicles for ovum pick-up. Theriogenology 1991; 35: 401-413. [Medline] [CrossRef]

11. Ferguson E, Bellows S, Lemieux F, Godke R. Development of a chute to facilitate transvaginal ultrasound guided oocyte aspiration (TUGA) in the sow. J Vet Med Sci 2013; 75: 191-194. [Medline] [CrossRef]

12. Diemunsch PA, Van Dorsselaer T, Torp KD, Schaeffer R, Geny B. Calibrated pneumoperitoneal venting to prevent $\mathrm{N}_{2} \mathrm{O}$ accumulation in the $\mathrm{CO}_{2}$ pneumoperitoneum during laparoscopy with inhaled anesthesia: an experimental study in pigs. Anesth Analg 2002; 94: 1014-1018. [Medline] [CrossRef] 